\title{
Tradução e adaptação semântica da Compulsive Buying Scale para o português brasileiro
}

\author{
Translation and semantic adaptation of the Compulsive \\ Buying Scale for Brazilian Portuguese
}

Priscilla Lourenço Leite', Bernard Pimentel Rangé', Rodolfo de Castro Ribas Junior², Tatiana Zambrano Filomensky ${ }^{3}$, Adriana Cardoso de Oliveira e Silva ${ }^{4}$

\section{RESUMO}

Objetivo: A Compulsive Buying Scale (Escala de Compras Compulsivas), instrumento breve e de fácil aplicação, apresenta como vantagem abarcar as principais dimensões do transtorno, sendo elas a compulsão pelas compras e o comportamento impulsivo. O presente estudo tem como objetivo a adaptação transcultural para o português do Brasil da Escala de Compras Compulsivas. Métodos: Para a etapa de adaptação semântica, dois psicólogos bilíngues e um tradutor fluente em português e inglês traduziram a escala de seu idioma de origem, o inglês, para o português. Em um segundo momento, foi realizada a retrotradução da escala por dois tradutores e um psicólogo. Finalmente, a escala foi aplicada em 20 participantes, de modo que pudessem ser feitos ajustes semânticos no instrumento em questão. Resultados: A colaboração entre profissionais especialistas em tradução bilíngue fluentes nos idiomas

\section{Palavras-chave}

Comportamento compulsivo, psicometria, escalas de graduação psiquiátrica, diagnóstico.

\section{Keywords}

Compulsive behavior, psychometrics, psychiatric status rating scales, diagnosis. inglês e português brasileiro, e psicólogos clínicos capacitados à avaliação sobre o constructo a ser mensurado, possibilitou o ajuste dos termos utilizados na versão final da escala para o idioma português, assegurando adequação semântica do instrumento. Assim, todos os itens obtiveram aprovação superior a 90\% em sua aplicação experimental. Conclusão: A versão da escala de compras compulsivas adaptada para o idioma português foi elaborada com êxito.

\section{ABSTRACT}

Objective: The Compulsive Buying Scale, a short and easy-to-apply instrument, has the advantage of comprising the main dimensions of the disorder, which are the compulsion for buying and the impulsive behavior. The present work aimed to adapt the scale transculturally to Brazilian Portuguese. Methods: To the step of semantic adaptation, a Portuguese-English Translator and two bilingual Psychologists translated the scale from English to Portuguese. Secondly, it was translated back to English for validation by two translators and a Psychologist. Finally, the scale was applied to 20 participants in order to perform semantic adjustments to the instrument. Results: The collaboration of professional translators and clinical psychologists, able to evaluate the quality of the material, made possible the adjustment of the text used in the final version of the scale in Portuguese, thus assuring semantic adequation. All items had approval of over $90 \%$ in the experimental application. Conclusion: The Brazilian Portuguese version of the Compulsive Buying Scale was successfully created.

1 Universidade Federal do Rio de Janeiro (UFRJ), Instituto de Psicologia (IP), Programa de Pós-graduação em Psicologia. 2 Universidade Federal do Rio de Janeiro (UFRJ), Instituto de Psicologia (IP), Departamento de Psicologia Geral e Experimental.

3 Faculdade de Medicina da Universidade de São Paulo (FMUSP), Instituto e Departamento de Psiquiatria, Programa Ambulatorial Integrado dos Transtornos do Impulsos.

4 Universidade Federal Fluminense (UFF), Laboratório de Tanatologia e Psicometria.

Endereço para correspondência: Priscilla Lourenço Leite Estrada do Pau-Ferro, 104, ap. 704 - 22743-051 - Rio de Janeiro, RJ Tel.: (21) 3327-7987 E-mail: priscilla.ufrj@gmail.com 


\section{INTRODUÇÃO}

O transtorno de compras compulsivas pode ser caracterizado como indivíduos que experimentam uma vontade irresistível, repetitiva e dominadora para comprar diversos itens'. Essa fissura é incontrolável e os pacientes só conseguem obter alívio da tensão por meio das compras excessivas. O ato de comprar é seguido de prazer, satisfação ou alívio; no entanto, essas sensações são seguidas de culpa, autorrecriminação e lamentação².

Originalmente descrito por Kraepelin, em 1915, como um impulso patológico para o ato de comprar, a oniomania costuma ser predominante no sexo feminino, com idades entre 18 e 30 anos, de classe média, tendo índice de prevalência pouco superior a $9 \%$ entre pacientes psiquiátricos internados $^{3}$. O problema tem sido encontrado, sobretudo, em países desenvolvidos e sua causa pode estar correlacionada ao alto nível de industrialização dos países em questão. Os principais problemas causados pelas compras compulsivas são: dívidas públicas, incapacidade em efetuar pagamentos, consequências jurídicas e financeiras, e sentimentos de culpa ${ }^{4}$. Alguns autores definiram o ato de comprar compulsivamente como tendo um caráter crônico, patológico e destrutivo, e ressaltaram ainda a dificuldade em interromper tal comportamento.

A Compulsive Buying Scale (Escala de Compras Compulsivas - CBS) pode ser amplamente utilizada ${ }^{5}$, pois seu dispositivo de triagem e diagnóstico do transtorno das compras compulsivas é altamente confiável, uma vez que distingue indíviduos que apresentam o transtorno da população normal. A CBS foi ainda traduzida para o alemão ${ }^{6}$, apresentando boa avaliação da tradução. Até o presente momento, no entanto, não foi validada aos parâmetros dessa população em questão. Estudos sobre a validação da CBS para a população Australiana ${ }^{7}$ obtiveram o Alpha de Cronbach 0.78. Eles realizaram os mesmos procedimentos estatísticos que a versão para o português utilizou para a validação dessa escala.

Por meio de uma revisão de literatura, foi possível observar que, apesar da pertinência e da importância do tema proposto neste trabalho, poucos estudos brasileiros aplacam o transtorno das compras compulsivas, assim como as formas de identificação, mensuração e diagnóstico, uma vez que não existem escalas validadas às normas semânticas e psicométricas do Brasil. Dessa forma, o principal objetivo deste estudo é a tradução e adaptação semântica da $\mathrm{CBS}^{8}$, para avaliação e diagnóstico do transtorno das compras compulsivas para o português do Brasil.

\section{MÉTODO}

A adaptação semântica da CBS foi realizada com autorização dos autores do instrumento original e seguiu os cinco passos propostos por Skevington?: 1) tradução inicial; 2) síntese das traduções; 3) tradução reversa; 4) revisão por especialistas e 5) aplicação piloto ou testagem preliminar.

A etapa de tradução $(T)$ contou com a participação de um tradutor e dois especialistas em saúde mental, ambos bilíngues. Os profissionais não tiveram contato entre si durante a execução dessa tarefa. As três propostas de tradução foram comparadas e as discrepâncias entre elas foram ajustadas.

Em seguida, na segunda fase, as propostas para itens geradas na etapa anterior foram comparadas com os itens que formam o instrumento original por outro especialista em saúde mental, gerando uma versão síntese (S). Na fase 3, a versão sintética formada foi retrotraduzida (RT) do português brasileiro para o idioma da escala original por três profissionais, sendo eles um tradutor e dois especialistas em saúde mental bilíngues, que não participaram das etapas anteriores. Discrepâncias foram ajustadas e a tradução reversa foi enviada, junto da versão em português brasileiro, para avaliação pelos autores da CBS para que eles pudessem avaliar eventuais alterações necessárias para a adaptação semântica ao contexto brasileiro.

$\mathrm{Na}$ etapa seguinte, cinco profissionais de saúde mental com experiência clínica atuaram como juízes avaliando os itens formulados quanto à clareza da linguagem, pertinência teórica e dimensão avaliada ${ }^{8,10}$. Havendo concordância com todos os aspectos, seguiu-se para a fase de aplicação experimental, em que o instrumento foi aplicado em 20 indivíduos, com diferentes níveis de escolaridade para avaliar o correto entendimento e aceitação dos itens propostos, sendo aceito o item mediante concordância superior a 80\%.

\section{Participantes}

Participaram da etapa de adaptação transcultural 20 indivíduos de uma população em geral, com níveis distintos de escolaridade e idade, de modo que fosse possível realizar ajustes na linguagem empregada na escala às normas semânticas e gramaticais do português brasileiro. Os participantes foram convocados através de e-mail. Todos eles concordaram com o termo de consentimento livre e esclarecido. A tabela 1 apresenta a descrição da amostra.

\section{Instrumentos}

O Termo de Consentimento Livre Esclarecido e o Questionário de Sociodemográficos foram os dois primeiros instrumentos aplicados. Além de um questionário de dados sociodemográficos, no qual foi possível fazer um levantamento da idade, sexo, estado civil, ocupação e nível de escolaridade, a CBS foi aplicada em sua versão pré-teste.

\section{CBS}

A CBS foi desenvolvida por Faber e O'Guinn ${ }^{8}$ em 1989, com intuito de mensurar a incidência do transtorno na população em geral e diagnosticar indivíduos com o transtorno das 
Tabela 1. Descrição da amostra

\begin{tabular}{|c|c|c|}
\hline Variável & $\mathrm{n}$ & $\%$ \\
\hline \multicolumn{3}{|l|}{ Sexo } \\
\hline Feminino & 11 & 55 \\
\hline Masculino & 9 & 45 \\
\hline \multicolumn{3}{|l|}{ Escolaridade } \\
\hline Ensino Fundamental Incompleto & 1 & 5 \\
\hline Ensino Fundamental Completo & 1 & 5 \\
\hline Ensino Médio Incompleto & 1 & 5 \\
\hline Ensino Médio Completo & 2 & 10 \\
\hline Ensino Superior Incompleto & 2 & 10 \\
\hline Ensino Superior Completo & 6 & 30 \\
\hline Técnico & 1 & 5 \\
\hline Pós-graduação Incompleta & 2 & 10 \\
\hline Pós-graduado(a) & 2 & 10 \\
\hline Mestrado & 1 & 5 \\
\hline Doutorado & 1 & 5 \\
\hline \multicolumn{3}{|l|}{ Idade (anos) } \\
\hline $18-29$ & 13 & 65 \\
\hline $30-39$ & 3 & 15 \\
\hline $40-69$ & 4 & 20 \\
\hline \multicolumn{3}{|l|}{ Estado Civil } \\
\hline Solteiro & 11 & 55 \\
\hline Casado & 7 & 35 \\
\hline Separado & 1 & 5 \\
\hline Divorciado & 1 & 5 \\
\hline Viúvo & 0 & 0 \\
\hline Outros & 0 & 0 \\
\hline
\end{tabular}

compras compulsivas. Os autores aplicaram uma escala em indivíduos que se autoproclamavam compradores compulsivos e participavam de um grupo de autoajuda para o comportamento compulsivo para comprar, localizados no Estado da Califórnia, Estados Unidos, e indivíduos da população geral, residentes no Estado de Illinois, Estados Unidos. Como critério de exclusão, retiraram de sua amostra participantes que já se encontravam em processo terapêutico, no intuito de não contaminar os dados da pesquisa. Dessa forma, a amostra final de participantes foi de 808 indivíduos.

O questionário inicial apresentava um conjunto de itens relacionados à compulsividade, retirados do subitem referente à psicastenia (transtorno obsessivo-compulsivo) do Inventário Multifásico de Personalidade de Minesotta (MMPI). Foram incluídas também medidas de materialismo, fantasia, sensações e sentimentos referentes ao ato de compras e consequências da compra compulsiva. Outros itens foram utilizados para avaliar a percepção e aspectos relacionados ao bem-estar econômico, sua relação com as dívidas, o comportamento de crédito e os sentimentos referentes ao processo de comprar.
A maioria dos itens foi avaliada por meio das escalas tipo Likert de 5 pontos. Por meio de autorrelato, os participantes deveriam declarar o nível de concordância ou discordância, para alguns itens da escala. Em outras questões, os entrevistados deveriam indicar a frequência ("muito frequentemente", "frequente", "às vezes", "raramente" ou "nunca") para mensurar o nível de engajamento comportamental e diferentes sentimentos experimentados com o ato de comprar.

De acordo com Faber e O'Guinn ${ }^{8}$, a pesquisa determinou uma série de fatores predisponentes ou correlatos associados a indivíduos que se identificaram como compradores compulsivos. Esses fatores incluem tanto aspectos psicológicos (atitudes e estados emocionais associados com o dinheiro e o ato de comprar, autoestima, compulsividade etc.) e aspectos econômicos (dívidas). Na construção da escala foram utilizados itens que estavam diretamente relacionados aos comportamentos, atitudes e emoções relacionados às compras.

Foram utilizados nessa construção 32 itens que avaliavam dimensões psicológicas, motivacionais e comportamentais da compra, por meio de pesquisas bibliográficas, senso comum e orientação de psicólogos e psiquiatras no que se referia à compulsão por compras. Por meio de um teste-piloto com um pequeno grupo de compradores compulsivos foi possível aperfeiçoar essas medidas ${ }^{11,12}$. Cada variável na análise discriminante apresentou uma diferença estatisticamente significativa $(p<0,10)$ sobre a medida da distância generalizada ( $\lambda$ (Lambda) de Wilk). Esse nível de significância estatística é aconselhável nos estágios iniciais da construção de um instrumento de mensuração.

A função discriminante derivada, por sua vez, classificou corretamente 320 das 372 pessoas nos estratos compulsivos $(86,02 \%)$ e 234 dos 270 nos estratos de comparação $(86,67 \%)$, para um total de $86,29 \%$ corretamente classificados. O instrumento de rastreamento utilizado na composição da CBS é composto pelo somatório das pontuações não ponderadas sobre os 14 itens selecionados na análise discriminante. Então, essa escala foi testada em uma população não clínica para a unidimensionalidade e confiabilidade. O exame de confiabilidade interna determinou que o alfa de Cronbach foi 0,83 . Este é considerado um nível aceitável, especialmente durante o desenvolvimento de uma nova escala.

A fim de fornecer uma validação parcial para a CBS, buscou-se comparar 16 indivíduos que apresentavam comportamentos compatíveis com os do transtorno de compras compulsivas, com participantes não clínicos. Foi utilizada uma série de testes-t para comparar as respostas dos dois grupos sobre as dimensões da escala. Não houve diferença estatisticamente significativa entre os compradores compulsivos e os membros da população em geral.

Outra forma utilizada no intuito de dar maior aporte à validade de construto foi considerar os valores médios do total da população em geral. O valor médio na medida de 
autoestima foi 14,92 para aqueles identificados como compradores compulsivos em comparação com 18,33 para a população em geral. Dessa forma, foi possível perceber que, em diversos aspectos, o grupo de compradores compulsivos apresenta diferenças ao ser comparado com o grupo não clínico, ou seja, os indivíduos selecionados da população em geral. Em conjunto, essas evidências sugeriram que a escala foi bem-sucedida em distinguir os indivíduos que possuem as mesmas atitudes psicológicas, econômicas, orientações de valor e comportamentos, tais como os compradores compulsivos e, portanto, parece possuir validade de construto.

Ao final, a função resultante da escala foi composta por 14 itens, a saber:

1. comprei coisas apesar de não conseguir pagar por elas;

2. senti que outros ficariam horrorizados se soubessem dos meus hábitos de compra;

3. se sobra algum dinheiro ao final do período de pagamento, tenho que gastá-lo;

4. paguei apenas o valor mínimo das minhas faturas de cartão de crédito;

5. comprei algo para me sentir melhor comigo mesmo;

6. emiti um cheque quando sabia que não tinha dinheiro suficiente no banco para cobri-lo;

7. só queria comprar coisas e não importa o que eu comprei;

8. eu sempre compro coisas simplesmente porque elas estão à venda:

9. senti-me ansioso ou nervoso em dias em que não fui às compras;

10. comprar é divertido;

11. senti-me deprimido após as compras;

12. comprei algo e quando cheguei em casa eu não sabia ao certo porque eu tinha comprado;

13. descontrolei-me ao comprar e não fui capaz de parar;

14. eu realmente acredito que se tivesse mais dinheiro resolveria a maioria dos meus problemas.

Ao final da validação, sete afirmativas permaneceram e atualmente compõem a escala:

1. se sobra algum dinheiro ao final do período de pagamento, tenho que gastá-lo;

2. senti que os outros ficariam horrorizados se soubessem dos meus hábitos de comprar;

3. comprei coisas apesar de não conseguir pagar por elas;

4. emiti um cheque quando sabia que não tinha dinheiro suficiente no banco para cobri-lo;

5. comprei algo para me sentir melhor comigo mesmo;

6. senti-me ansioso ou nervoso em dias em que não fui às compras;

7. paguei apenas o valor mínimo das minhas faturas de cartão de crédito.

\section{Procedimentos}

Esta pesquisa foi aprovada pelo Comitê de Ética em Pesquisa da Escola de Enfermagem Anna Nery/Hospital-Escola São
Francisco de Assis EEAN/HESFA, da Universidade Federal do Rio de Janeiro (UFRJ), protocolo n. 102/2010. Todos os participantes concordaram com o Termo de Consentimento Livre Esclarecido, após terem sido apresentados à pesquisa e aos procedimentos envolvidos no estudo.

OTermo de Consentimento Livre Esclarecido e o questionário de dados pessoais foram os dois primeiros instrumentos dos protocolos, nessa ordem, por conterem os dados iniciais, apresentação da pesquisa e convite à participação. Em seguida, o indivíduo deveria responder se concordavam com a estrutura semântica e linguagem aplicada no conjunto de 7 afirmativas da CBS. Caso discordassem, existia uma lacuna na qual poderiam sinalizar mudanças na frase.

\section{RESULTADOS}

$\mathrm{Na}$ avaliação da composição da amostra de respondentes, foi observado que 55\% eram mulheres, com nível de escolaridade superior, ou seja, 30\% apresentavam ensino superior completo. Quanto à estado civil, 55\% dos participantes responderam ser solteiros e as idades obtiveram a média de 30,4 anos. Esses dados, embora representem indivíduos de uma população em geral, sem o diagnóstico para o transtorno das compras compulsivas, são similares aos dos estudos epidemiológicos acerca dessa desordem.

Para a confecção final da escala, a tabela 2 apresenta as etapas 1 a 4 do processo de adaptação semântica da CBS e as mudanças semânticas e reformulações das afirmativas.

\section{DISCUSSÃO}

Ao avaliarmos o processo de tradução da escala de compras compulsivas, observamos as versões dos itens. O primeiro item, inicialmente, apresentava em seu enunciado o verbo "ter" relacionando-se à ideia de que o indivíduo avaliado, necessariamente, para apresentar o comportamento de comprar compulsivamente necessite ter dinheiro a mais do que sua renda sobrando ao final do mês. Em sua versão final, o enunciado do item foi ajustado para a versão original da escala, que pondera apenas sobre sobrar algum dinheiro ao final do mês.

No item 2, sua primeira tradução considerou que o fator preponderante para avaliação sobre o comportamento da compra compulsiva estaria relacionada à forma como os "outros" percebem o comportamento. Na síntese desse item, foi observada a composição original do enunciado em questão, que se refere à forma como o sujeito avaliado se sente em relação ao seu comportamento em adquirir. Assim, em sua versão final, foi utilizado o verbo "sentir", sugerido pelos juízes especialistas. 
Tabela 2. Etapas da tradução inicial, síntese, tradução reversa e versão final em português brasileiro para os itens da Escala de Compras Compulsivas

\begin{tabular}{|c|c|c|c|}
\hline \multicolumn{4}{|c|}{ Escala de Compras Compulsivas (CBS) } \\
\hline Tradução & Sintese & Tradução reversa & Versão final \\
\hline $\begin{array}{l}\text { Se eu tiver algum dinheiro sobrando ao final } \\
\text { do mês, eu preciso gastá-lo }\end{array}$ & $\begin{array}{l}\text { Se sobra algum dinheiro ao final do período } \\
\text { de pagamento, tenho que gastá-lo }\end{array}$ & $\begin{array}{l}\text { If there is any money left at the end of the } \\
\text { month I must spend it }\end{array}$ & $\begin{array}{l}\text { Se sobrar algum dinheiro no final do mês, eu } \\
\text { tenho que gastá-lo }\end{array}$ \\
\hline $\begin{array}{l}\text { Se as pessoas soubessem o quanto gasto, } \\
\text { ficariam horrorizadas }\end{array}$ & $\begin{array}{l}\text { Senti que outros ficariam horrorizados se } \\
\text { soubessem dos meus hábitos de comprar }\end{array}$ & $\begin{array}{l}\text { I feel others could be shocked if they knew } \\
\text { about my shopping habits }\end{array}$ & $\begin{array}{l}\text { Senti que os outros poderiam ficar } \\
\text { horrorizados se soubessem dos meus hábitos } \\
\text { de comprar }\end{array}$ \\
\hline $\begin{array}{l}\text { Eu compro coisas mesmo que não possa } \\
\text { pagá-las }\end{array}$ & $\begin{array}{l}\text { Comprei coisas apesar de não conseguir } \\
\text { pagar por elas }\end{array}$ & I buy things even when I can't afford them & $\begin{array}{l}\text { Comprei coisas apesar de não conseguir } \\
\text { pagar por elas }\end{array}$ \\
\hline $\begin{array}{l}\text { Já passei um cheque mesmo sabendo que } \\
\text { não tinha dinheiro suficiente no banco para } \\
\text { cobri-lo }\end{array}$ & $\begin{array}{l}\text { Emiti um cheque quando sabia que não tinha } \\
\text { dinheiro suficiente no banco para cobri-lo }\end{array}$ & $\begin{array}{l}\text { I write checks knowing that I have } \\
\text { insufficient funds in my bank account to } \\
\text { honor them }\end{array}$ & $\begin{array}{l}\text { Emiti um cheque mesmo sabendo que não } \\
\text { tinha dinheiro suficiente no banco para } \\
\text { cobri-lo }\end{array}$ \\
\hline Comprei algo para me sentir melhor & Comprei algo para me sentir melhor comigo & I buy things to make myself feel good & $\begin{array}{l}\text { Comprei algo para me sentir melhor comigo } \\
\text { mesmo }\end{array}$ \\
\hline $\begin{array}{l}\text { Me senti nervoso ou ansioso nos dias em que } \\
\text { não comprei algo }\end{array}$ & $\begin{array}{l}\text { Me senti ansioso ou nervoso em dias em que } \\
\text { não fui às compras }\end{array}$ & $\begin{array}{l}\text { I feel anxious or nervous when I spend a day } \\
\text { without buying something }\end{array}$ & $\begin{array}{l}\text { Me senti ansioso ou nervoso em dias em que } \\
\text { não fui às compras }\end{array}$ \\
\hline $\begin{array}{l}\text { Paguei apenas o mínimo das faturas do meu } \\
\text { cartão de crédito }\end{array}$ & $\begin{array}{l}\text { Paguei apenas o valor mínimo das minhas } \\
\text { faturas de cartão de crédito }\end{array}$ & $\begin{array}{l}\text { I only pay the minimum amount due of my } \\
\text { credit card invoices }\end{array}$ & $\begin{array}{l}\text { Paguei apenas o valor mínimo das faturas do } \\
\text { meu cartão de crédito }\end{array}$ \\
\hline
\end{tabular}

No item 3, inicialmente traduzido para "eu compro coisas mesmo que não possa pagá-las", os primeiros juízes consideraram o enunciado mal estruturado, podendo gerar confusões de ordem semântica. Assim, na etapa de síntese, o item foi modificado para "comprei coisas apesar de não conseguir pagar por elas". O corpo de especialistas considerou esse item satisfatório, sendo essa sua versão final.

Para composição do quarto item da escala, a tradução inicial considerou o termo "wrote a check" do idioma de origem da escala como "já passei um cheque". Ao ser avaliado pelo corpo de juízes, foi considerado o valor semântico original. Assim, o enunciado do item ficou "emiti um cheque quando sabia que não tinha dinheiro suficiente no banco para cobri-lo". Para sua versão final, os especialistas avaliaram a utilização do tempo verbal "saber", buscando uma concordância mais apropriada. Assim, em sua versão final, o item adquiriu a seguinte estrutura: "emiti um cheque mesmo sabendo que não tinha dinheiro suficiente no banco para cobri-lo".

Com relação ao item 5, não houve mudanças significativas em seu processo de tradução e adaptação, e, em sua versão final, foi considerada a frase: "Comprei algo para me sentir melhor comigo mesmo". No que se refere à adaptação do item 6, os juízes trocaram a frase "me senti nervoso ou ansioso nos dias em que não comprei algo" para "me senti ansioso ou nervoso em dias em que não fui às compras", no intuito de aproximar a tradução de sua versão original no idioma inglês "felt anxious or nervous on days I didn 't go shopping".

Para o item 7, a tradução inicial conferiu o seguinte enunciado: "paguei apenas o valor mínimo das faturas do meu cartão de crédito". Na versão de síntese, o corpo de juízes considerou acrescentar a palavra "valor", de modo que ficasse mais claro que a afirmativa referia-se a um pagar a fatura do cartão, sem quitar sua dívida. Assim, em sua versão final, o item 7 obteve a seguinte estrutura: "paguei apenas o valor mínimo das faturas do meu cartão de crédito".
Após as etapas de tradução da escala de compras compulsivas, foi solicitada participação de 20 respondentes, para a etapa de aplicação experimental. A tabela 3 apresenta o resultado dessa etapa. A escala de compras compulsivas obteve porcentagem superior a $90 \%$ em todos os itens, indicando que ela estaria apta para aplicação na população em geral.

\section{CONCLUSÃO}

Diversos pesquisadores concordam que os compradores compulsivos têm uma obsessão (preocupação) com a compra e uma compulsão que os leva a compras excessivas e repetitivas ${ }^{13,14}$. O desejo de comprar pode fazer o indivíduo a adquirir um produto, independente de sua necessidade, no intuito de amenizar sua vontade. Essa compra não planejada e desnecessária pode estar relacionada a uma falta de controle dos impulsos, caracterizando a compra impulsiva.

Sendo comprar uma atividade considerada gratificante, inócua, agradável e, sobretudo, socialmente reconhecida e aceitável, sendo vista antigamente como uma atividade exercida por poucos, só os que possuíam poder aquisitivo elevado, o transtorno das compras compulsivas pertence à categoria das "new addictions"15.

A CBS é um instrumento voltado especificamente para mensuração de sinais e sintomas relacionados ao transtorno de compras compulsivas, apresentando bons parâmetros psicométricos em sua versão original. Sendo breve e de fácil aplicação, é importante ferramenta para avaliação clínica quanto à prevenção do transtorno ou sua evolução ao longo do tempo. Também possui aplicação em pesquisas, podendo colaborar como instrumento em estudos de prevalência do transtorno na população geral.

Os demais instrumentos já existentes para mensuração e diagnóstico da compra compulsiva apresentam algumas 
Tabela 3. Índices de concordância observados na aplicação da versão pré-teste da Escala para Compras Compulsivas

\begin{tabular}{|c|c|c|c|c|c|c|c|}
\hline \multirow{2}{*}{ Participantes } & \multicolumn{7}{|c|}{ Escala para Compras Compulsivas } \\
\hline & Item 1 & Item 2 & Item 3 & Item 4 & Item 5 & Item 6 & Item 7 \\
\hline a1 & 1 & 1 & 1 & 1 & 1 & 1 & 1 \\
\hline a2 & 1 & 1 & 1 & 1 & 1 & 1 & 1 \\
\hline a3 & 1 & 1 & 1 & 1 & 1 & 1 & 1 \\
\hline a4 & 1 & 1 & 1 & 1 & 1 & 1 & 1 \\
\hline a5 & 1 & 1 & 1 & 0 & 1 & 1 & 1 \\
\hline a6 & 1 & 1 & 1 & 1 & 1 & 0 & 1 \\
\hline a7 & 1 & 1 & 1 & 1 & 1 & 1 & 1 \\
\hline a8 & 1 & 0 & 1 & 1 & 1 & 1 & 0 \\
\hline a9 & 1 & 1 & 1 & 0 & 1 & 1 & 1 \\
\hline $\mathrm{a} 10$ & 1 & 1 & 1 & 1 & 1 & 1 & 1 \\
\hline a11 & 1 & 1 & 1 & 1 & 1 & 1 & 1 \\
\hline a12 & 1 & 1 & 1 & 1 & 1 & 0 & 1 \\
\hline a13 & 1 & 1 & 1 & 1 & 1 & 1 & 0 \\
\hline a14 & 1 & 0 & 1 & 1 & 1 & 1 & 1 \\
\hline a15 & 1 & 1 & 1 & 1 & 1 & 1 & 1 \\
\hline a16 & 1 & 1 & 1 & 1 & 1 & 1 & 1 \\
\hline a17 & 1 & 1 & 1 & 1 & 1 & 1 & 1 \\
\hline a18 & 1 & 1 & 1 & 1 & 1 & 0 & 1 \\
\hline a19 & 1 & 1 & 1 & 1 & 1 & 1 & 1 \\
\hline a 20 & 1 & 1 & 1 & 1 & 1 & 1 & 1 \\
\hline Total & 20 & 18 & 20 & 18 & 20 & 17 & 18 \\
\hline Porcentagem & 100 & 90 & 100 & 90 & 100 & 95 & 90 \\
\hline
\end{tabular}

restrições ${ }^{16}$. A Compulsive-buying Measurement Scale ${ }^{14}$ e a Compulsive-buying Scale de DeSarbo \& Edwards ${ }^{17}$ centram-se em aferir apenas a dimensão do transtorno de controle dos impulsos. A Yale-Brown Obsessive-Compulsive Scale-Shopping Version ${ }^{18}$ (Y-BOCS-SV) verifica a severidade da compra compulsiva, sob o aspecto dimensional do transtorno obsessivo-compulsivo, e o Questionnaire about buying behavior ${ }^{19}$ avalia apenas as consequências financeiras da compra compulsiva.

A partir da adaptação da CBS para o português do Brasil, pretende-se aplacar a deficiência de escalas de avaliação e diagnóstico do transtorno do comprar compulsivo. Este estudo procurou atender a essa demanda, no que concerne ao aumento da compreensão acerca do transtorno, buscando alternativas diagnósticas, de mensuração, prevenção e tratamento dessa desordem.

\section{REFERÊNCIAS}

1. Lejoyeux M, Haberman N, Solomon J, Adès J. Comparison of buying behavior in depressed patients presenting with or without compulsive buying. Compr Psychiatry. 1999;40(1):51-6.

2. McElroy SL, Keck Jr P, Pope Jr H, Smith JM, Strakowski S. Compulsive buying: a report of 20 cases. J Clin Psychiatry. 1994;55(6):242-8.

3. Grant JE, Levine L, Kim D, Potenza MN. Impulse control disorders in adult psychiatric inpatients. Am J Psychiatry. 2005;162(11):2184-8.

4. Black D. A review of compulsive buying disorder. World Psychiatry. 2007;6(1):14-8.

5. Müller A, Mitchell J. Compulsive buying: clinical foundations and treatment. In: Taylor and Francis Group. New York (NY): Routledge; 2011.
6. Mueller A, Mitchell JE, Mertens C, Mueller U, Silbermann A, Burgard M, et al. Comparison of treatment seeking compulsive buyers in Germany and the United States. Behav Res Ther. 2007;45(7):1629-38.

7. Phau I, Woo C. Understanding compulsive buying tendencies among young Australians: The roles of money attitude and credit card usage. Marketing Intelligence \& Planning. 2008;26(5):441-58.

8. Faber RJ, O'Guinn TC. Classifying compulsive consumers: advances in the development of a diagnostic tool. Association for consumer research. 1989;16:738-44.

9. Skevington SM, Bradshaw J, Saxena S. Selecting national items for the WHOQOL: conceptual and psychometric considerations. Soc Sci Med. 1999;48(4):473-87.

10. Faber RJ, Christenson GA. In the mood to buy: differences in the mood states experienced by compulsive buyers and other consumers. Psychology and Marketing. Special Issue: Aberrant Consumer Behavior. 1996:13:803-20.

11. Pasquali L. Escalas psicométricas. In: Pasquali L (org.). Instrumentação Psicológica: fundamentos e práticas. Porto Alegre: Artmed; 2010. p.116-35.

12. Pasquali L (Org.) Técnicas de exame psicológico - TEP. Manual. Vol. I: Fundamentos das técnicas psicológicas. São Paulo: Casa do Psicólogo; 2001.

13. Hirschman EC. The consciousness of addiction: toward a general theory of compulsive consumption. Journal of Consumer Research, 1992:19:155-79.

14. Kyrios M, Frost RO, Steketee G. Cognitions in compulsive buying and acquisition. Cog Ther Res. 2004;28:241-58.

15. Guerreschi C. New addictions: as novas dependências. São Paulo: Paulus; 2007.

16. Ridgway NM, Kukar-Kinney M, Monroe KB. An expanded conceptualization and a new measure of compulsive buying. J Consum Res. 2008:35:622-39.

17. D'Astous A. An inquiry into the compulsive side of normal consumers. J Consum Pol. 1990:13:1531.

18. DeSarbo WS, Edwards EA. Typologies of compulsive buying behavior: A constrained clusterwise regression approach. J Consum Psychol. 1996:5:231-62.

19. Monahan P, Black DW, Gabel J. Reliability and validity of a scale to measure change in persons with compulsive buying. Psychiatry Res. 1996:64:59-6. 